Vol. 7, No. 1, 2017

\title{
FEATURES OF DISTRIBUTION OF THREE-DIMENSIONAL QUASI- STATIONARY ELECTROMAGNETIC FIELD IN A SYSTEM WITH PLANAR INTERFACE BETWEEN MEDIA
}

\author{
Yuriy Vasetsky, Iryna Mazurenko, Konstantin Dziuba \\ Institute of Electrodynamics, Ukrainian National Academy of Sciences \\ yuriy.vasetsky@gmail.com
}

(C) Vasetsky Yu., Mazurenko I., Dziuba K., 2017

\begin{abstract}
It is established that the distribution of density of induced current in conducting half-space has no component perpendicular to the planar interface between media, regardless of (1) the properties of the medium, (2) configuration of a current-carrying contour, and (3) the current dependence on time. It is shown that the surface density of electric charge is determined only by the normal component of the strength of the induced electric field of the source-system of currents.

In the case of a strong skin effect in electric conductive medium, conclusions have been drawn based on the correct solution of the task of electromagnetic field. Spreading the statement on the general case of medium with arbitrary electro physical properties is based on the well-known zero solution of the boundary problem for a vertical component of electric field strength in electrically conductive medium defined as a task of homogeneous equation of parabolic type with zero boundary conditions. Results are illustrated by the example of calculating the surface density of the electric charge in the case of the planar current-carrying contour if the current is supplied using two parallel conductors perpendicular to the central part of the contour.
\end{abstract}

Key words: quasi-stationary three-dimensional electromagnetic field, arbitrary loop with current, eddy currents.

\section{Introduction}

In many technical applications, the mathematical model in which primary current sources are alternating currents flowing along the contours of certain configuration is used for determining the distribution of electromagnetic field $[1,2,3]$. When the primary currents flow near conducting bodies, eddy currents are induced in them, and these currents also participate in the formation of the field. The eddy currents can perform useful work in technological processes, for example, in induction heating devices $[4,5]$ or in devices for magnetic-pulse sheet pressing. In some other cases, the external fields can cause harm to control and monitoring units, as well as the service staff, for example, in electrohydraulic pulse installations [6].

In $[7,8]$, the problem for the case of planar interface between conducting and dielectric media is considered for quasi-stationary electromagnetic field in general formulation. However, the analytical solution has been obtained for vector potential and induction of the magnetic field only in a dielectric domain, where the current-carrying contour was located. Besides, the neglect of displacement current did not allow us to determine the electric field strength in the case of spatial loops. The result has been found only for planar contours, which were parallel to the interface between media.

In general, the three-dimensional analytical solution of quasi-stationary problem in the system "arbitrary contour with alternating current - conducting half-space" in the whole space does exist. The aim of this work consists in ascertaining the general peculiarities of distribution of electric field strength and that of current density in conducting medium and, as a consequence, determining the vertical component of electric field strength at the interface between media and determining the field sources at this planar surface.

\section{Mathematical model}

Let us consider an arbitrary contour in an electrically non-conductive non-magnetic medium with the relative dielectric permittivity $\varepsilon_{e}$ and let the current $I_{0}(t)$ flow along the contour. The contour is located near the conducting body whose relative magnetic permeability is $\mu$ (in Fig. 1, original current-carrying contour is shown by a solid line in the upper half-space $z>0$ ).

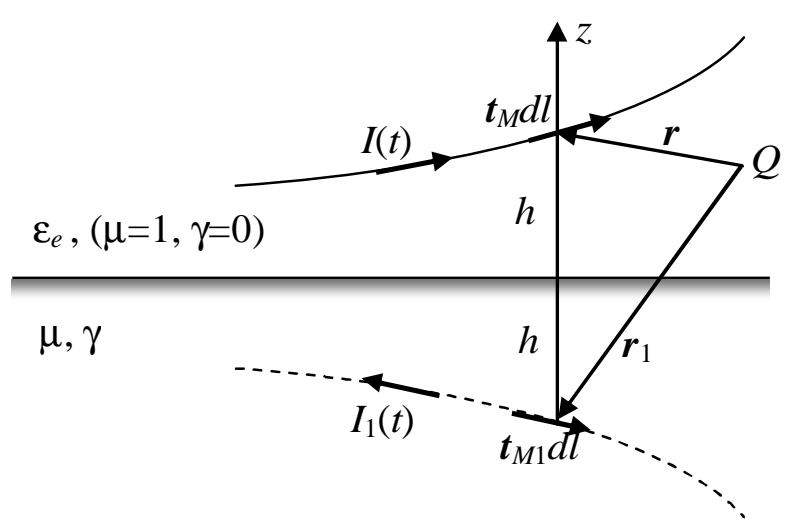

Fig. 1. Computational model. 
It is assumed that the dimensions of the contour are considerably less than the size of the planar patch of the body surface; this enables us to use the model of a current-carrying contour located above the conducting half-space. We consider the linear problem of calculation of quasi-stationary electromagnetic field concerning the case when the current density satisfies the condition of continuity ( $\operatorname{div} j=0$ ) and, therefore, the currentcarrying contour is closed.

Defining the problem as quasi-stationary assumes the satisfaction of two restrictions: firstly, the wavelength $\lambda$ of the electromagnetic field must be considerably longer than any characteristic size $L$ of the electromagnetic system, i.e. $\lambda=2 \pi / \omega \sqrt{\mu \mu_{0} \varepsilon \varepsilon_{0}}>L$; secondly, in the equation of total current the density of displacement current $j_{D}$ is to be neglected in comparison with the density of the conduction current ${ }^{\prime} j[9,10]$.

The problem is described by Maxwell's equations for such vectors of field as electric field strength ${ }^{\prime}$, magnetic field strength $H$, magnetic induction $B$ and electric displacement $D$; the density of the current from external sources $j_{0}$ in contour elements [9] being taken into account:

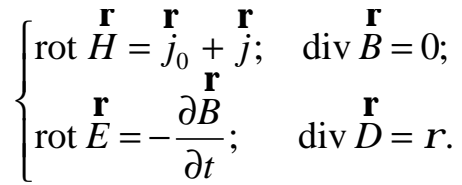

For the linear problem, the free charge $\rho$ in piecewise continuous medium can be located only at the interface between media with the corresponding surface density $\sigma$.

The constitutive equations, which complement equations (1), are:

$$
\begin{array}{ll}
z>0: & \dot{D}_{e}=\varepsilon_{e} \varepsilon_{0} \dot{E}_{e}, \dot{B}_{e}=\mu_{\mathrm{r}} \dot{H}_{e}, \\
z<0: & j_{i}=\gamma E_{i}, B_{i}=\mu \mu_{0} H_{i},
\end{array}
$$

where physical quantities in the domains $z>0$ and $z<0$ are marked with the subscripts " $e$ " and " $i$ " respectively.

On the surface of the conductor, the boundary conditions for the tangential and normal components of the vectors of electromagnetic field are satisfied. Moreover, the condition of field vanishing at infinity must be established:

$$
\begin{gathered}
\left\{\stackrel{r}{e_{z}} \times\left(\stackrel{r}{E^{+}}-\stackrel{r}{E^{-}}\right)=0, \stackrel{r}{e_{z}} \times\left(\stackrel{r}{H^{+}}-\stackrel{r}{H}^{-}\right)=0 .\right. \\
\left\{\stackrel{r}{e_{z}} \cdot \stackrel{r}{E^{-}}=0, \stackrel{r}{e_{z}} \cdot \stackrel{r}{E^{+}}=\sigma / \varepsilon_{e} \varepsilon_{0}, \stackrel{r}{e_{z}} \cdot\left(\stackrel{r}{B^{+}}-\stackrel{r}{B^{-}}\right)=0 .\right.
\end{gathered}
$$

Here, the field vectors on the interface between the media located on the positive and negative parts of the axis $z$ have subscripts "+" and "-", respectively (Fig. 1), $e_{z}$ is the unit vector in the direction of the axis $z$.

Let us introduce a vector potential $A$ and a scalar potential $\varphi$ :

$$
\stackrel{r}{B}=\operatorname{rot} \stackrel{r}{A} ; \quad \stackrel{r}{E}=-\operatorname{grad} \phi-\frac{\partial \dot{A}}{\partial t}
$$

and let us use the Coulomb gauge:

$$
\operatorname{div} \stackrel{\prime}{A}=0 \text {. }
$$

If in quasi-stationary approximation the displacement current are neglected, Maxwell's equations for the potentials take on a form as follows

$$
\Delta A=-\mu \mu_{0}\left[j_{0}+\gamma\left(-\operatorname{grad} \phi-\frac{\partial A}{\partial t}\right)\right], \Delta \phi=-\frac{\rho}{\varepsilon \varepsilon_{0}} .
$$

Here, in the right side of the equation for the vector potential, the density of conduction current is put in parentheses. In the upper half-space only the source current flows

$$
\dot{J}_{0}=I_{0} \delta\left(\stackrel{r}{r_{M}}-\stackrel{r}{r_{Q}}\right) \stackrel{I}{t}_{M}
$$

where $\delta\left(\stackrel{r}{r}_{M}-\stackrel{r}{r}_{Q}\right)$ is the Dirac delta function, $\stackrel{\prime}{t}_{M}$ is the unit vector of tangent to the contour line. In the conducting half-space $z<0$ the eddy current flows. The charge is distributed along the interface between the media

$$
\rho=\sigma(x, y) \delta(z) .
$$

The equation (7) describes the known fact that in application of Coulomb gauge, under the conditions of considering the field in all the space and its absence at infinity, the vector potential is determined by the conduction current, and the scalar potential is determined by the electric charges $[11,12]$.

Note that when we use only vector potential, the electric field strength in (5) can be determined with the accuracy to within a potential summand. However, the equation of scalar potential also contains the unknown value of the surface density of charge $\sigma$, that does not allow us to formulate the boundary-value problem for scalar potential and to determine the potential component of electric field strength. The authors of the work [13] pay attention to this property showing that first of all the difficulty arises for three-dimensional problems, and the solution becomes unambiguous if additional conditions are set. The ambiguity in determining the strength of the electric field is not essential, for example, in the determination of the 
induction of the magnetic field or e.m.f. induced in a closed contour. However, the calculation of electric field strength becomes necessary, for example, if local characteristics of action of electric field or energy flux density of electromagnetic field $\stackrel{P}{P}=\stackrel{E}{E} \times \stackrel{H}{H}$ are determined and in some other cases.

The main task of this article is to show that the features of field distribution and current density in conducting media in the case of planar interface between dielectric and conducting media enable us to solve the aforesaid problem, i.e., to find the boundary conditions for electric field strength and determine the surface charge density.

3. Peculiarities of current distribution and conditions on the interface between media.

Let us, first, consider the problem for strong skin effect and then generalize the results for the general case of arbitrary electrophysical properties of conducting medium.

\subsection{Strong skin effect.}

In the case of strong skin effect, for the calculation of the field outside the conducting body, it is necessary to solve the corresponding stationary problem for perfectly conducting body of the same shape [7, 8]. Under a given distribution of current density $\dot{j}_{0}$ of the sources, the statement of the boundary value problem is reduced to defining Maxwell's equation for the magnetic field strength $\stackrel{1}{H}$ and to the condition of the absence of the normal component of the field on the surface:

$$
\operatorname{rot} \stackrel{\prime}{H}=\stackrel{\prime}{j}, \quad \operatorname{div} \stackrel{\prime}{H}=0, \quad \stackrel{\prime}{H} \cdot \stackrel{r}{n}=0,
$$

where $n$ is the unit vector outer normal to the surface of the conducting body.

The boundary value problem (10) completely determines the existence of a unique solution.

For the analysis of the electromagnetic field in superficial layer of conducting body, the known model [9] of the diffusion of the planar field into conductive half-space usually holds true. In such a model, the initial physical quantity is the tangential component $\dot{H}_{\tau}$ of the field, whose local value for an arbitrarily shaped body is determined from the solution of outer problem (1). The surface density $\dot{j}_{s}$ of current in a conducting body is determined by the value of tangential component $\dot{H}_{\tau}$ and must satisfy the condition of continuity:

$$
\dot{j}_{s}=\stackrel{r}{n} \times \stackrel{\text { ' }}{H}, \quad \operatorname{div} \dot{j}_{s}=0 .
$$

The solution to the problem (10) for the planar interface between media is the magnetic field above the surface of the conducting body for $z>0$, which is caused by the current $I_{0}$ of the original contour and by the fictitious current $I_{1}$ of the mirror contour (Fig. 1) [16, 17].

In Fig. 1 the directions of elements $t d l$ of the original contour and $t_{1} d l$ of the mirror contour are characterized by unit tangent vectors $t$ and $t_{1}, d l$ is the length of elements of the contour. Projections of tangent vectors onto the vertical axis are equal in absolute values and opposite in their directions $\left(t_{1 z}=-t_{z}\right)$, and the projections $\quad t_{\|}$and $t_{1 \|}$ onto the plane of the interface between media are equal in their lengths and directions $t_{1 \|}=t_{\|}$, i.e., $t=t_{z} e_{z}+\dot{t}_{\|}, t_{1}=t_{\|}-t_{z} e_{z}$.

The magnetic field strength $\dot{H}$ created by two current-carrying contours in an arbitrary point $(z>0)$ is the following

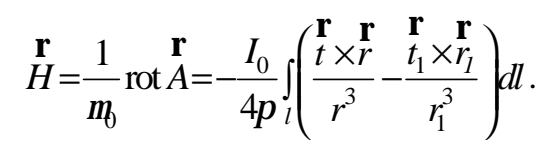

Taking into consideration the fact that in the case when the vectors connecting the point of observation $Q$ located on the planar interface between media with the elements ${ }^{t} d l$ and $t_{1} d l$ are $r=\stackrel{t}{c}+h \dot{e}_{z}$, the magnetic field strength can be presented in terms of coordinates of the original contour only in the following form

$$
\stackrel{r}{H}(z=0)=\frac{I_{0}}{2 \pi} \stackrel{r}{e_{z}} \times \int_{l} \frac{e_{z} \times(t \times r)}{r^{3}} d l .
$$

From (13), it immediately follows that the normal component of the field strength is absent, $\stackrel{1}{H} \cdot \stackrel{r}{e_{z}}=0$. This indicates that (13) is the solution of the stated problem (10) for the planar interface between media.

Subsequently, the surface density of current is found from (11):

$$
\stackrel{r}{j_{s}}=\stackrel{r}{e_{z}} \times\left[-\frac{I_{0}}{2 \pi} \int_{l} \frac{t^{\prime} \times \stackrel{r}{r}}{r^{3}} d l\right]=\frac{2}{\mu_{0}} \underset{e_{z}}{r} \times \operatorname{rot} \stackrel{r}{A_{0}},
$$

where $\stackrel{\prime}{A}_{0}$ is the vector potential of the field created by the original current.

According to knowledge of secondary sources, there is a surface electric current flowing on the interface between media, and the electric charge with definite surface density $\sigma$ can be distributed there. All the sources, namely, the original current $I_{0}$, surface current density $\dot{j}_{s}$ and distributed surface charge $\sigma$ together make up the electric field in the whole space including the domain $z<0$, where the total field is equal to zero. A simple analytical solution to the considered problem 
enables us to obtain a clear idea about the formation of both secondary sources creating the field.

So far we have considered the magnetic field above the surface of the conducting body for $z>0$, to determine which it was sufficient to introduce a fictitious current of the image contour. In this case, the vector potential is represented by the formula

$$
\stackrel{r}{A}=\stackrel{r}{A_{0}}+\stackrel{r}{A_{1}}=\frac{\mu_{0} I_{0}}{4 \pi} \int\left(\frac{\mathrm{I}}{l}-\frac{\mathrm{I}_{1}}{r}\right) d l .
$$

In fact, besides the original contour, the current flows only on the surface of the conducting body.

The density of the surface current does not contain the component perpendicular to the interface between media. As a result, the magnetic vector potential of the whole system of currents in the whole space including the domain $z<0$ is

$$
\stackrel{r}{A^{\prime}}=\stackrel{r}{A_{0}}+\stackrel{r}{A_{s}}=\frac{\mu_{0} I_{0}}{4 \pi} \int_{l} \frac{t}{r} d l+\frac{\mu_{0}}{4 \pi} \int_{S} \frac{\dot{j}_{s}}{r} d S .
$$

As $\dot{A}_{s}(z=0) \cdot \stackrel{r}{e}_{z}=0$, at the interface between media the normal component of the vector potential ${ }^{\prime} A_{z}^{\prime}='^{\prime}(z=0) \cdot \stackrel{r}{e}_{z}=\stackrel{'}{A}_{0 z}$ is not equal to zero. Note that because of continuity of the vector potential, i.e., $\operatorname{div} A^{\prime}=0$, the normal component $A_{o z}$ does not change crossing the interface between media, i.e., $A_{o z}^{+}=A_{o z}^{-}$.

The scalar potential $\varphi_{\sigma}$ and the related potential component $\stackrel{\prime}{E}_{\sigma}=-\operatorname{grad} \phi_{\sigma}$ of the electric field strength are caused by electric charges which (in the considered problem) are located on the interface between media with the surface density $\sigma$ :

$$
\varphi_{\sigma}=\frac{1}{4 \pi \varepsilon_{e} \varepsilon_{0}} \int_{S} \frac{\sigma}{r} d S .
$$

The normal components of the electric field strength, which is caused by the surface charges on opposite sides of the interface, are equal in absolute value and have opposite signs:

$$
\hat{E}_{\sigma z}^{+}=-\hat{E}_{\sigma z}^{-} .
$$

Besides, on the interface the vertical component of the field created by the electric charges is compensated by the strength of the induced electric field, i.e.,

$$
-\frac{\partial A_{0 z}}{\partial t}+E_{\sigma z}^{-}=0 .
$$

The surface density $\sigma$ of electric charge is determined from the boundary condition
$\frac{\sigma}{\varepsilon_{0}}=E_{\sigma z}^{+}-E_{\sigma z}^{-}$. Taking into account (18) and (19), we finally have:

$$
\frac{\sigma}{\varepsilon_{e} \varepsilon_{0}}=E_{z}^{+}=-2 \frac{\partial A_{0 z}}{\partial t}
$$

Here $E_{z}^{+}=E_{\sigma z}^{+}-\frac{\partial A_{0 z}}{\partial t}$ is the total strength of the electric field on the interface of media.

3.2. General case: arbitrary properties of conducting medium.

Now, let us consider the general case of the conducting medium. Let us show that also in this case for a circuit with a contour of arbitrary spatial configuration the vertical components of electrical field strength and induced current density are equal to zero. From Maxwell's equations (1), it follows that the electric field strength in conducting medium satisfies the parabolic homogeneous equation:

$$
\Delta \stackrel{r}{\mathrm{E}}-\mu \mu_{0} \gamma \frac{\partial \stackrel{\prime}{E}}{\partial t}=0
$$

The vertical component of electric field strength at the interface between the conducting and dielectric media, according to the first boundary condition (4) is equal to zero. At infinitely distant points, this component of electric field is also equal to zero. As a result, for the vertical component of electric field in the domain $z<0$, we obtain the task for the parabolic homogeneous equation with zero boundary conditions:

$$
\left\{\begin{array}{l}
\Delta E_{z}-\mu \mu_{0} \gamma \frac{\partial E_{z}}{\partial t}=0, \\
E_{z}(z=0-0)=0, E_{z}(\infty)=0 .
\end{array}\right.
$$

The solution of this problem for a steady-state process "without initial conditions" for a half-infinite domain has a unique zero solution [18]. The field will be also absent at any instant of time under zero initial conditions.

Thus, in a conducting medium not only in the case of strong skin effect, but also in the case of any properties of the conducting medium and under any dependence on time of the original current flowing along the spatial contour, the components of electric field strength and current density perpendicular to the planar interface between media are equal to zero.

Without repeating the consideration which enabled us to obtain the relationship (20), let us note that the absence of vertical components of density of the induced current (now in the whole half-space) has the corollary of the relations perfectly analogical to those of (20) for electric field strength and for surface density of electric 
charge at the planar interface. To determine the corresponding values at the interface between the media, it is sufficient to know only vertical component of the induced electrical field of the original current $\frac{\partial A_{0 z}}{\partial t}$.

Besides, on the basis of superposition principle, the result holds true for any system of closed currents.

Note that the surface density of electric charge and, consequently, the vertical component of electric field strength are conditioned by the presence of segments of the contour perpendicular to the planar surface of the conducting body. Therefore, when they are absent, namely, when the original currents flow along planar contours situated in parallel to the interface between media, the electric field strength has only a vertical component, and for the calculation of this component, it is sufficient to know only the distribution of the vector potential of the electromagnetic field.

Finally, we present an example of the calculation of surface density of electric charge in a system where central part of current-carrying contour is located in parallel to the planar surface of the conducting body. The current is supplied by two parallel conductors oriented perpendicularly to the central part of the contour (Fig. 2). As the distribution of electric charge is determined only by segments of the contour perpendicular to the interface between the media, in this example the distribution of charge will be the same for any configuration of the central part of the circuit.

For the chosen geometry of the contour, the vertical component of the vector potential and, correspondingly, the surface charge density can be presented by means of simple algebraic expressions.

$$
\begin{aligned}
& \sigma=-2 \frac{\varepsilon_{e} \varepsilon_{0} \mu_{0}}{4 \pi} \frac{\partial I_{0}}{\partial t} \int_{h}^{\infty}\left[\left(z_{M}^{2}+\rho_{2}^{2}\right)^{-1 / 2}-\left(z_{M}^{2}+\rho_{1}^{2}\right)^{-1 / 2}\right] d z_{M}= \\
& =\frac{\varepsilon_{e} \varepsilon_{0} \mu_{0}}{2 \pi} \frac{\partial I_{0}}{\partial t} \ln \frac{h+\left(h^{2}+\rho_{2}^{2}\right)^{1 / 2}}{h+\left(h^{2}+\rho_{1}^{2}\right)^{1 / 2}}
\end{aligned}
$$

Here $h$ is the distance from the central part of the contour to the plane interface of media; $\rho_{1,2}^{2}=\left(x_{Q}-x_{1,2}\right)^{2}+\left(y_{Q}-y_{1,2}\right)^{2}$, where $\left(x_{1}, y_{1}\right)$ and $\left(x_{2}, y_{2}\right)$ are coordinates on the planar surface of the location of two vertical conductors along which the current is directed to the central part of the contour and from it, respectively.

The results of the calculation according to (23) are shown in Fig. 2 in the form of lines $\sigma / \sigma_{m}=$ const, where $\sigma_{m}$ is the maximal value of surface density of the distributed electric charge.

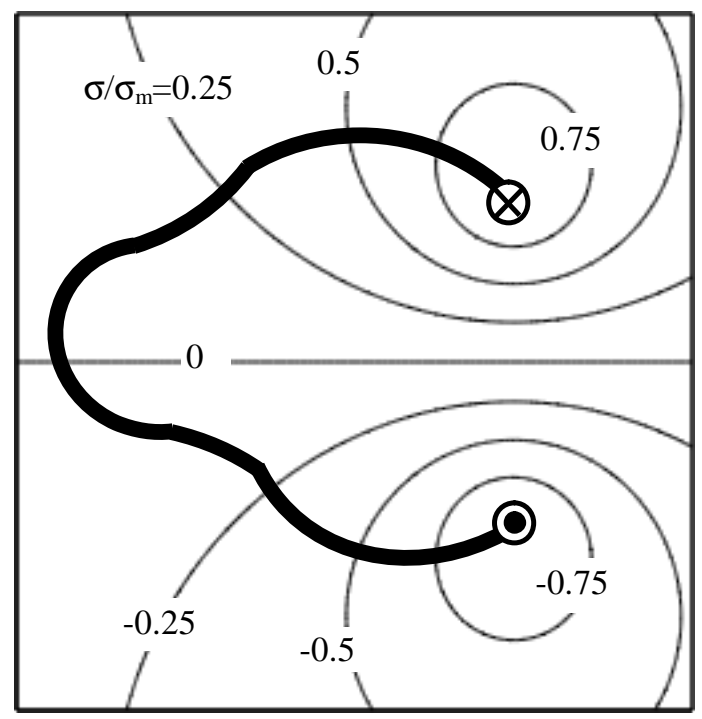

Fig. 2. Distribution of surface density of electric charge.

\section{Conclusions}

In quasi-stationary formulation of the problem for the systems with planar interface between dielectric and conducting media, there is no component of electric field strength perpendicular to the interface and no such a component of current density in the conducting half-space. The result holds true for any spatial distribution of the initial system of non-stationary currents. On the media interface, the surface density of electric charge and the vertical component of electric field strength in dielectric medium are determined only by the normal component of the induced electric field of the initial system of currents.

\section{References}

[1] Yu. Vasetsky, Asymptotic methods for solving electrodynamics problems in systems with bulky curvilinear conductors, Kyiv, Ukraine: Naukova dumka, 2010. (Russian)

[2] J. Acero, R. Alonso, J. Burdio, L. Barragan, and D. Puyal, "Analytical Equivalent Impedance for a Planar Induction Heating System", IEEE Transaction on Magnetics, vol. 42, no. 1, pp. 84-86, 2006.

[3] G. Tsitsikyan, "Electromagnetic field of a linear conductor with a current parallel to a boundary interface "air medium - conducting half-space"" Elektrichestvo, no. 12, pp. 55-61, 1997. (Russian)

[4] V. Rudnev, D. Loveless, R. Cook, and M. Black, Handbook of induction heating, Marcel Dekker Inc., 2003.

[5] I. Kondratenko and A. Rashchepkin, "Induction heating of moving stripe by current-carrying contours", Tekhnichna Elektrodynamika, no. 3, pp. 3-9, Kyiv, Ukraine: Institute of Electrodynamics of Ukraine, 1999. (Russian)

[6] Yu. Batygin, N. Lavinskyi, and L. Khimenko, Pulsed magnetic fields for progressive technologies. Kharkiv, Ukraine: Most-Tornado, 2003. (Russian) 
[7] Yu. Vasetsky, Electromagnetic field of impulse current that flows above conducting half-space, Kyiv, Ukraine: Institute of Electrodynamics, 1992. (Russian)

[8] Yu. Vasetsky, L. Gorodzha, and I. Mazurenko, "Approximate model for the calculation of alternative magnetic field of arbitrary contour taking into account eddy current in conducting half-space", Tekhnichna Elektrodynamika, no. 1, pp. 88-93, Kyiv, Ukraine: Institute of Electrodynamics of Ukraine, 1999. (Russian)

[9] K. Polivanov, Theoretical bases of electrical engineering. No. 3. The theory of electromagnetic field. Moscow, Russia: Energiya, 1969. (Russian)

[10] K. Shimoni, Theoretical electrical engineering Moscow, Russia: Mir, 1964. (Russian)

[11] I. E. Tamm, Bases of the theory of electricity, Moscow, Russia: GITTL, 1956. (Russian)

[12] A. Fedorchenko The theoretical physics. Classical electrodynamics, Kyiv, Ukraine: Vyshcha shkola, 1988. (Russian)

[13] O. Tozoni and I. Maergoyz, Calculation of threedimensional electromagnetic fields, Kyiv, Ukraine: Tekhnika, 1974. (Russian).)

[14] L. Landau and E. Lifshits, Electrodynamics of continua. Moscow, Russia: Nauka, 1982. (Russian)

[15] H. Knoepfel, Pulsed High Magnetic Fields, Amsterdam-London: North-Holland Publishing Company, 1970.

[16] Yu. Vasetskiy "The electromagnetic field of a spatial loop with a current above a planar surface of a conducting body with a strong skin-effect", Elektrichestvo, no 3, pp. 55-61. (Russian)

[17] Y. M.Vasetsky and D.I.Vlasov, "On magnetic field determination of a current-carrying contour above the planar surface of a perfect electrical conductor", Tekhnichna Elektrodynamika, no. 2, pp. 9-10, Kyiv, Ukraine: Institute of Electrodynamics of Ukraine, 2012. (Russian)

[18] A. Tihonov and A. Samarskiy, Equations of mathematical physics. Moscow, Russia: Nauka, 1966. (Russian)

\section{ОСОБЛИВОСТІ РОЗПОДІЛУ ТРИВИМІРНОГО КВАЗІСТАЦІОНАР- НОГО ЕЛЕКТРОМАГНІТНОГО ПОЛЯ В СИСТЕМІ 3 ПЛОСКОЮ ГРАНИЦЕЮ РОЗПОДІЛУ СЕРЕДОВИЩ}

Юрій Васецький, Ірина Мазуренко, Костянтин Дзюба

Встановлено, що розподіл густини індукованого струму в електропровідному півпросторі не має компоненти, що перпендикулярна плоскій границі поділу середовищ незалежно від властивостей середовища, конфігурації контуру вихідного струму в діелектричному півпросторі й залежності струму від часу. Показано, що поверхнева густина електричного заряду визначається тільки нормальною компонентою напруженості індукованого електричного поля вихідного струму контура.

У випадку сильного скін-ефекту в електропровідному півпросторі висновок зроблено на основі точного рішення задачі про електромагнітне поле. Поширення ствердження на загальний випадок середовища 3 довільними електрофізичними властивостями засновано на відомому нульовому рішенні крайової задачі для вертикальної компоненти напруженості електричного поля в електропровідному середовищі, яка сформульована як задача для однорідного рівняння параболічного типу 3 нульовими крайовими умовами. Результати проілюстровано на прикладі розрахунку поверхневої густини електричного заряду у разі плоского контуру, коли підвід струму здійснюється по двом паралельним провідникам, що перпендикулярні центральній частини контуру.

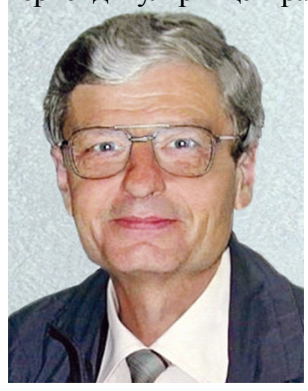

Yuriy Vasetsky - graduated from Moscow Power Engineering Institute, Russia (1973). He was awarded Ph.D. Degree (1981); D.Sc. Degree (1995) in Theoretical Electrical Engineering, and the title of Professor (2007)

Leading research scientist of the Institute of Electrodynamics, National Academy of Sciences of Ukraine;

Prof. of National Aviation University, Department of Theoretical and Applied Physics.

Author of more than 150 scientific papers in the fields of electrodynamics and electrophysics of electromagnetic systems. Author of the book on asymptotic methods of electrodynamics problems and a manual on electrodynamics. The results and methods are used for the development of technological equipment, high-current magnetic systems, high voltage equipment

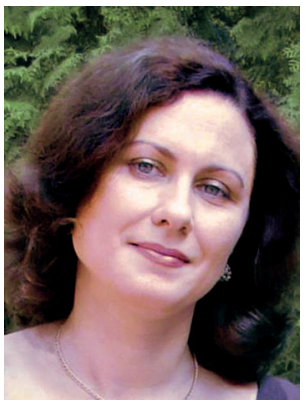

Iryna Mazurenko - graduated from Kyiv National Technical University (1996); she was awarded Ph.D. in Theoretical Electrical Engineering in 2003.

Senior research scientist of Theoretical Electrical Engineering Department, Institute of Electrodynamics, National Academy of Sciences of Ukraine.

Author of about 50 scientific papers in the fields of modeling of power systems elements, mathematical modeling of electromagnetic processes in induction heating devices

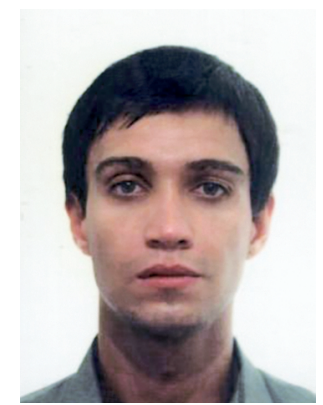

Konstantin Dzjuba graduated from National Aviation University, Ukraine, as Electrical Engineer (2008).

$\mathrm{He}$ conducts researches in electrical engineering, transfer of power, impulse technology, and he is the author of several papers in these scientific fields. 